TRANSACTIONS OF THE

AMERICAN MATHEMATICAL SOCIETY

Volume 350, Number 9, September 1998, Pages 3797-3814

S 0002-9947(98)02211-9

\title{
HOMOCLINIC SOLUTIONS AND CHAOS IN ORDINARY DIFFERENTIAL EQUATIONS WITH SINGULAR PERTURBATIONS
}

\author{
JOSEPH GRUENDLER
}

\begin{abstract}
Ordinary differential equations are considered which contain a singular perturbation. It is assumed that when the perturbation parameter is zero, the equation has a hyperbolic equilibrium and homoclinic solution. No restriction is placed on the dimension of the phase space or on the dimension of intersection of the stable and unstable manifolds. A bifurcation function is established which determines nonzero values of the perturbation parameter for which the homoclinic solution persists. It is further shown that when the vector field is periodic and a transversality condition is satisfied, the homoclinic solution to the perturbed equation produces a transverse homoclinic orbit in the period map. The techniques used are those of exponential dichotomies, Lyapunov-Schmidt reduction and scales of Banach spaces. A much simplified version of this latter theory is developed suitable for the present case. This work generalizes some recent results of Battelli and Palmer.
\end{abstract}

\section{INTRODUCTION}

In this work we shall consider differential equations which take the equivalent forms

$$
\begin{aligned}
\epsilon \dot{x} & =f_{0}(x)+\epsilon f_{1}(x, \epsilon, t), \\
\dot{x} & =f_{0}(x)+\epsilon f_{1}(x, \epsilon, \epsilon t)
\end{aligned}
$$

with $x \in \mathbb{R}^{n}, \epsilon \in \mathbb{R}$.

We make the following assumptions about (1):

(i) $f_{0}$ and $f_{1}$ are $\mathcal{C}^{3}$ in all arguments.

(ii) $f_{0}(0)=f_{1}(0, \epsilon, t)=0$.

(iii) The eigenvalues of $D f_{0}(0)$ lie off the imaginary axis.

(iv) The unperturbed equation has a homoclinic solution. That is, there exists a differentiable function $t \rightarrow \gamma(t)$ such that $\lim _{t \rightarrow-\infty} \gamma(t)=\lim _{t \rightarrow+\infty} \gamma(t)=0$ and $\dot{\gamma}(t)=f_{0}(\gamma(t))$.

(v) $f_{1}$ and its derivatives are bounded in $(x, \epsilon)$ uniformly in $t$.

Received by the editors December 28, 1995 and, in revised form, October 23, 1996.

1991 Mathematics Subject Classification. Primary 34E15, 34C37, 58F13.

Key words and phrases. Ordinary differential equations, homoclinic solutions, bifurcations, singular perturbations.

This work supported in part by the J. William Fulbright Commission. 
In (1b) we make the change of variable $\tau=t-\alpha / \epsilon$ and then change back to $t$ to obtain

$$
\dot{x}=f_{0}(x)+\epsilon f_{1}(x, \epsilon, \epsilon t+\alpha) .
$$

The advantage in (2) is the presence of the additional parameter $\alpha$.

The present work was motivated by recent results of Battelli and Palmer [1]. They consider equations similar to (1) but with a coefficient of $\epsilon^{2}$ for $f_{1}$. Our objective is to find conditions on $f_{1}$ such that (2) has a transverse homoclinic orbit for $\epsilon \neq 0$. We use the method of Lyapunov-Schmidt to obtain a bifurcation function $(\epsilon, \beta, \alpha) \rightarrow M(\epsilon, \beta, \alpha)$ where $\epsilon$ and $\alpha$ are as in (2). The vector $\beta$ represents directions, other than that provided by $\dot{\gamma}$, tangent to $T_{P} W^{s} \cap T_{P} W^{u}$ where $P=\gamma(0)$ and $W^{s}, W^{u}$ denote the stable, unstable manifolds respectively for the equilibrium of the unperturbed equation. $M$ is linear in $\epsilon$ and quadratic in $\beta$ with coefficients obtained as Melnikov integrals. The coefficient of $\epsilon$ is a nonlinear function of $\alpha$.

If $M\left(\epsilon_{0}, \beta_{0}, \alpha_{0}\right)=0$, then (2) has a homoclinic solution when $\epsilon=s^{2} \epsilon_{0}$ for sufficiently small $s \in \mathbb{R}$. Furthermore if, in addition, $D_{(\beta, \alpha)} M\left(\epsilon_{0}, \beta_{0}, \alpha_{0}\right)$ is nonsingular, the homoclinic orbit is transversal.

In the literature on homoclinic bifurcations it is usually assumed that the unperturbed equation has a hyperbolic equilibrium with stable, unstable manifolds which meet in dimension one. This is the case in [1]. Some work where the manifolds are allowed to meet in dimension two are [9], [11], [12]. For the case of regular perturbations, a general theory for manifolds which meet in arbitrary dimension was developed in [5], [6], [7]. Here, we extend this general theory to the singularly perturbed case thus providing a generalization of [1]. A second improvement over that work is a simplification of the use of scales of Banach spaces.

In the present case a certain difficulty is encountered in the usual Banach space techniques. If one starts with a space of functions, $\mathbb{Z}$, with a prescribed rate of exponential decay at $t= \pm \infty$ and uses (2) to define $F: \mathbb{Z} \times \mathbb{R} \times \mathbb{R} \rightarrow \mathbb{Z}$ by

$$
F(\varphi, \epsilon, \alpha)(t)=f_{0}(\varphi(t))+\epsilon f_{1}(\varphi(t), \epsilon, \epsilon t+\alpha),
$$

then $F$ is not differentiable with respect to $\epsilon$ due to the term $\epsilon t D_{3} f_{1}(\varphi(t), \epsilon, \epsilon t+\alpha)$ which appears in the derivative.

Battelli and Palmer deal with this difficulty by introducing a scale of Banach spaces consisting of functions with small exponential growth. A basic reference for this idea is Vanderbauwhede and Van Gils [13]. An integral part of this theory is a sophisticated form of the contraction mapping theorem which uses simultaneously an infinite family of Banach spaces.

We show in our work how it is possible, in the present case, to reduce the problem to a single element in the family of Banach spaces and then use the standard contraction mapping theorem. This results in a simpler proof than in [1].

Here we thank Michal Fečkan for his many comments, corrections and suggestions as well as his encouragement and motivation. Thanks also to the referee for additional comments and references.

\section{Exponential Dichotomies and Scales of Banach Spaces}

We begin with the linear equation $\dot{u}=A(t) u$ which will serve below as the variational equation along $\gamma$. The following result is Theorem 2 in [6] with a slight change of notation. The idea for the proof of the following theorem is illustrated in the case $n=2$ on pp. 214-215 of [12]. 
1. Theorem. Let $t \rightarrow A(t)$ be a matrix-valued function continuous for $t \in \mathbb{R}$. Suppose there exists a constant matrix, $A_{0}$, and a scalar $b>0$ such that

$$
\sup _{t}\left|A(t)-A_{0}\right| e^{b|t|}<\infty
$$

Suppose also the eigenvalues of $A_{0}$ satisfy $|\Re(\lambda)| \geq 3 a$ for some $a>0$. Then there exists a fundamental solution, $U$, for the differential equation $\dot{u}=A(t) u$ along with a constant $K_{0}>0$ and four projections $P_{s s}, P_{s u}, P_{u s}, P_{u u}$ such that $P_{s s}+P_{s u}+P_{u s}+P_{u u}=I$ and such that the following hold:

(i) $\left|U(t)\left(P_{s s}+P_{s u}\right) U(s)^{-1}\right| \leq K_{0} e^{2 a(t-s)}$ for $t \leq s \leq 0$,

(ii) $\left|U(t)\left(P_{u u}+P_{u s}\right) U(s)^{-1}\right| \leq K_{0} e^{2 a(s-t)}$ for $s \leq t \leq 0$,

(iii) $\left|U(t)\left(P_{s s}+P_{u s}\right) U(s)^{-1}\right| \leq K_{0} e^{2 a(s-t)}$ for $0 \leq s \leq t$,

(iv) $\left|U(t)\left(P_{u u}+P_{s u}\right) U(s)^{-1}\right| \leq K_{0} e^{2 a(t-s)}$ for $0 \leq t \leq s$.

Furthermore, there exists an integer $d \geq 0$ such that $\operatorname{rank}\left(P_{s s}\right)=\operatorname{rank}\left(P_{u u}\right)=d$.

In this notation the first subscript denotes exponential decay, "s", or exponential growth, "u", at $-\infty$. The second subscript is the same for $+\infty$. Explicit examples of this theorem can be found in [5], [6] and [7] as well as below.

In the language of dichotomies, see Coppel [3], we see that Theorem 1 provides a two-sided exponential dichotomy. For $t \leq 0$ an exponential dichotomy is given by the fundamental solution $U$ and the projection $P_{u u}+P_{u s}$ while for $t \geq 0$ such is given by $U$ and $P_{s s}+P_{u s}$. Note that if $d=0$ then $P_{s s}=P_{u u}=0$ and there exists a single exponential dichotomy valid for all $t$.

We shall let $u_{j}$ denote column $j$ of $U$ and assume that these are numbered so that

$$
P_{u u}=\left[\begin{array}{ccc}
I_{d} & 0_{d} & 0 \\
0_{d} & 0_{d} & 0 \\
0 & 0 & 0
\end{array}\right], \quad P_{s s}=\left[\begin{array}{ccc}
0_{d} & 0_{d} & 0 \\
0_{d} & I_{d} & 0 \\
0 & 0 & 0
\end{array}\right]
$$

Here, $I_{d}$ denotes the $d \times d$ identity matrix, $0_{d}$ the $d \times d$ zero matrix. We also let $u_{i}^{\perp}$ denote row $i$ of $U^{-1}$.

If $U^{\perp}$ is the matrix with $u_{j}^{\perp}$ in column $j$, then $U^{\perp t}=U^{-1}$. Differentiating $U U^{\perp t}=I$ we obtain $\dot{U} U^{\perp t}+U \dot{U}^{\perp t}=0$ so that $\dot{U}^{\perp}=-\left(U^{-1} \dot{U} U^{\perp t}\right)^{t}=$ $-A(t)^{t} U^{\perp}$. Thus, $U^{\perp}$ is a fundamental solution for the adjoint equation. The functions $\left\{u_{1}^{\perp}, \ldots, u_{d}^{\perp}\right\}$ are a basis for the vector space of bounded solutions to the adjoint equation while the functions $\left\{u_{d+1}, \ldots, u_{2 d}\right\}$ are the same for the original equation.

Let $U, P_{u u}, a$ be as in Theorem 1. For each $\eta, 0<\eta \leq a$, we define the Banach spaces

$$
\begin{aligned}
& \mathbb{Z}_{\eta}=\left\{z \in \mathcal{C}^{0}\left(\mathbb{R}, \mathbb{R}^{n}\right): \sup _{t}|z(t)| e^{-\eta|t|}<\infty\right\}, \\
& \overline{\mathbb{Z}}_{\eta}=\left\{z \in \mathbb{Z}_{\eta}: \int_{-\infty}^{\infty} P_{u u} U(t)^{-1} z(t) d t=0\right\},
\end{aligned}
$$


with norm the supremum in the definition. We also define $\mathbb{Z}_{0}=\bigcap_{\eta>0} \mathbb{Z}_{\eta}$. Next, define the variation of constants map $K: \overline{\mathbb{Z}}_{\eta} \rightarrow \mathbb{Z}_{\eta}$ by

$$
\begin{aligned}
(K z)(t)=U(t)\left[-\int_{0}^{\infty} P_{s u} U(s)^{-1} z(s) d s\right. & +\int_{0}^{t}\left(P_{s s}+P_{s u}\right) U(s)^{-1} z(s) d s \\
& \left.+\int_{-\infty}^{t}\left(P_{u s}+P_{u u}\right) U(s)^{-1} z(s) d s\right] \\
=U(t)\left[\int_{-\infty}^{0} P_{u s} U(s)^{-1} z(s) d s \quad\right. & +\int_{0}^{t}\left(P_{s s}+P_{u s}\right) U(s)^{-1} z(s) d s \\
& \left.-\int_{t}^{\infty}\left(P_{s u}+P_{u u}\right) U(s)^{-1} z(s) d s\right] .
\end{aligned}
$$

In this definition, the first and second forms are intended for $t \leq 0$ and $t \geq 0$, respectively.

Now consider the nonhomogeneous equation

$$
\dot{z}=A(t) z+h
$$

with $A$ as in Theorem 1. If $h \in \overline{\mathbb{Z}}_{\eta}$ then $K h$ is a solution in $\mathbb{Z}_{\eta}$ for (3) satisfying $\left\langle(K h)(0), u_{j+d}^{\perp}(0)\right\rangle=0$ for $1 \leq j \leq d$ and the general solution in $\mathbb{Z}_{\eta}$ is

$$
z=K h+\sum_{j=1}^{d} \beta_{j} u_{j+d}
$$

for constants $\beta_{j}$.

Next, let $\Phi: \mathbb{R} \rightarrow \mathbb{R}$ be a smooth function satisfying $\sup _{t}\left|\Phi(t) u_{j}(t)\right|<\infty$ for all $j$ and $\int_{-\infty}^{\infty} \Phi(t) d t=1$. Then define a projection $\Pi: \mathbb{Z}_{\eta} \rightarrow \mathbb{Z}_{\eta}$ by

$$
\begin{aligned}
(\Pi z)(t) & =\Phi(t) \int_{-\infty}^{\infty} U(t) P_{u u} U(s)^{-1} z(s) d s \\
& =\Phi(t) \sum_{i=1}^{d} u_{i}(t) \int_{-\infty}^{\infty}\left\langle u_{i}^{\perp}(s), z(s)\right\rangle d s .
\end{aligned}
$$

It is easy to check that $\Im(I-\Pi)=\overline{\mathbb{Z}}_{\eta}$ and

$$
\int_{-\infty}^{\infty}\left\langle u_{i}^{\perp}, \dot{z}-A z\right\rangle d t=0 \quad \text { for } z \in \mathbb{Z}_{\eta}, \quad 1 \leq i \leq d,
$$

so that

$$
\Pi(\dot{z}-A z)=0 .
$$

For details in a similar case see [6].

Now consider (3) for $h \in \mathbb{Z}_{\eta}$. Using $\Pi$ we decompose the problem of solving for $z \in \mathbb{Z}_{\eta}$ into

$$
\begin{aligned}
& \dot{z}=A(t) z+(I-\Pi) h, \\
& \Pi h=0 .
\end{aligned}
$$

We can solve the first of these for $z \in \mathbb{Z}_{\eta}$. The second of these becomes our bifurcation equation.

Our technique for obtaining a transversality condition will be to show that for the homoclinic solution when $\epsilon \neq 0$ the variational equation satisfies $d=0$ in the terminology of Theorem 1. Our next result will be used for this purpose. 
We consider equations of the form $\dot{u}=(A(t)+S(t)) u$ where $t \rightarrow S(t)$ is a bounded matrix-valued function. $S$ defines a function $\hat{S}: \mathbb{Z}_{\eta} \rightarrow \mathbb{Z}_{\eta}$ defined by the formula $(\hat{S} z)(t)=S(t) z(t)$ with $\|\hat{S}\| \leq \sup _{t}|S(t)|$. The following result is proved in $[7]$.

2. Theorem. Let $\dot{x}=A x$ be as in Theorem 1 along with $U, a, d$ and the four projections $P_{s s}, P_{s u}, P_{u s}, P_{u u}$. Let $t \rightarrow S(t)$ be a bounded matrix-valued function continuous for $t \in \mathbb{R}$. Suppose that $\|\hat{S}\|$ is sufficiently small so that $I-K(I-\Pi) \hat{S}$ is invertible. Define a $d \times d$ matrix $\mathcal{F}(S)$ by

$$
\mathcal{F}(S)_{i j}=\int_{-\infty}^{\infty}\left\langle u_{i}^{\perp}, \hat{S}[I-K(I-\Pi) \hat{S}]^{-1} u_{j+d}\right\rangle d t, \quad 1 \leq i, j \leq d .
$$

If $\mathcal{F}(S)$ is nonsingular, then the differential equation $\dot{u}=(A(t)+S(t)) u$ has no nonzero solutions which decay at both $\pm \infty$ so that $d=0$ in the terminology of Theorem 1.

\section{Perturbation Theory}

We now turn our attention to (2). Henceforth we shall let $U, d, P_{s s}, P_{s u}, P_{u s}$, $P_{u u}$ be the corresponding quantities obtained by applying Theorem 1 to the variational equation $\dot{u}=D f_{0}(\gamma) u$. In addition to the conventions mentioned following Theorem 1 we shall assume $u_{2 d}=\dot{\gamma}$. This is always possible since, as a solution to the variational equation which decays at both $\pm \infty, \dot{\gamma}$ can be expressed as a linear combination of columns $u_{d+1}$ through $u_{2 d}$ of $U$ and a linear change of coordinates among these columns will not affect the projections.

In (2) we make the change of variable $x=\gamma+z$. The equation for $z$ is

$$
\dot{z}(t)=D f_{0}(\gamma(t)) z(t)+g(z(t), \epsilon, \alpha, t)
$$

where

$$
g(x, \epsilon, \alpha, t)=f_{0}(\gamma(t)+x)-f_{0}(\gamma(t))-D f_{0}(\gamma(t)) x+\epsilon f_{1}(\gamma(t)+x, \epsilon, \epsilon t+\alpha) .
$$

We wish to convert (6) to an integral equation by solving for $z$ as in (3). The problem is that for $z \in \mathbb{Z}_{\eta}$ we have no control over the growth of $g(z(t), \epsilon, \alpha, t)$. To correct this we introduce the so-called cut-off function [13]. Let $\chi: \mathbb{R}^{n} \rightarrow \mathbb{R}$ denote a smooth function satisfying

$$
\chi(x)= \begin{cases}1, & \text { if }|x| \leq 1 \\ 0, & \text { if }|x| \geq 2 .\end{cases}
$$

For each $\rho>0$ define $g_{\rho}: \mathbb{R}^{n} \times \mathbb{R} \times \mathbb{R} \times \mathbb{R} \rightarrow \mathbb{R}^{n}$ by $g_{\rho}(x, \epsilon, \alpha, t)=g(x, \epsilon, \alpha, t) \chi(x / \rho)$.

For future reference we note:

$$
\begin{aligned}
& g_{\rho}(0,0, \alpha, t)=0, \\
& D_{1} g_{\rho}(0,0, \alpha, t)=0, \\
& D_{11} g_{\rho}(0,0, \alpha, t)=D^{2} f_{0}(\gamma(t)), \\
& D_{2} g_{\rho}(0,0, \alpha, t)=f_{1}(\gamma(t), 0, \alpha) .
\end{aligned}
$$

We also define $G_{\rho}: \mathbb{Z}_{\eta} \times \mathbb{R} \times \mathbb{R} \rightarrow \mathbb{Z}_{0} \subset \mathbb{Z}_{\eta}$ by

$$
G_{\rho}(z, \epsilon, \alpha)(t)=g_{\rho}(z(t), \epsilon, \alpha, t) .
$$


We now replace (6) with the equation

$$
\dot{z}=D f_{0}(\gamma) z+G_{\rho}(z, \epsilon, \alpha) .
$$

Note that if $z$ is a solution to (8) with $|z(t)| \leq \rho$ for all $t$, then $z$ is a solution to (6). This idea plays a role in the proof of Theorem 4.

3. Lemma. Given $\delta>0$ there exist $\rho_{0}>0, \epsilon_{0}>0$ such that

$$
\left|D_{1} g_{\rho_{0}}(x, \epsilon, \alpha, t)\right|<\delta
$$

for $|\epsilon| \leq \epsilon_{0}$.

Proof. Differentiating the definition of $g$ we get

$$
D_{1} g(x, \epsilon, \alpha, t)=D f_{0}(\gamma(t)+x)-D f_{0}(\gamma(t))+\epsilon D_{1} f_{1}(\gamma(t)+x, \epsilon, \epsilon t+\alpha) .
$$

By hypothesis (v) for $(1), D_{1} f_{1}$ is bounded in $(x, \epsilon)$ uniformly in $t$, and, hence, $D_{1} g$ is bounded uniformly in $(\alpha, t)$.

Define $A=\sup _{x}|D \chi(x)|, B=\sup _{t} \sup _{|x| \leq 1}\left|D^{2} f_{0}(\gamma(t)+x)\right|$ and let $\delta>0$ be given. We can choose $\epsilon_{1}$ and $\rho_{0}$ satisfying $\epsilon_{1}>0,0<\rho_{0} \leq \min \left\{\frac{1}{2}, \frac{\delta}{8 A B}\right\}$ such that

$$
\left|D_{1} g(x, \epsilon, \alpha, t)\right| \leq \frac{\delta}{2} \quad \text { if }|x| \leq 2 \rho_{0},|\epsilon| \leq \epsilon_{1} .
$$

Applying Taylor's theorem (see $\S 8.14$ of Dieudonné [4]) to the function $\phi(s)=$ $f_{0}(\gamma(t)+s x)$ we have, if $|x| \leq 2 \rho_{0}$,

$$
\begin{array}{r}
\left|f_{0}(\gamma(t)+x)-f_{0}(\gamma(t))-D f_{0}(\gamma(t)) x\right|=\left|\phi(1)-\phi(0)-\phi^{\prime}(0)\right|=\left|\int_{0}^{1}(1-s) \phi^{\prime \prime}(s) d s\right| \\
=\left|\int_{0}^{1}(1-s) D^{2} f_{0}(\gamma(t)+s x) x x d s\right| \leq 2 B \rho_{0}^{2} \leq \frac{\delta \rho_{0}}{4 A} .
\end{array}
$$

Since, by hypothosis, $D_{1} f_{1}(x, \epsilon, t)$ is bounded uniformly in $t$, we can choose $\epsilon_{2}>0$ such that

$$
\left|\epsilon f_{1}(\gamma(t)+x, \epsilon, \epsilon t+\alpha)\right| \leq \frac{\alpha \rho_{0}}{4 A} \quad \text { if }|\epsilon| \leq \epsilon_{2},|x| \leq 2 \rho_{0} .
$$

Define $\epsilon_{0}=\min \left\{\epsilon_{1}, \epsilon_{2}\right\}$. Combining these results we have $|g(x, \epsilon, \alpha, t)| \leq \frac{\delta \rho_{0}}{2 A}$ if $|\epsilon| \leq \epsilon_{0},|x| \leq 2 \rho_{0}$.

When $|x| \leq 2 \rho_{0}$ and $|\epsilon| \leq \epsilon_{0}$ we now have

$$
\begin{aligned}
\left|D_{1} g_{\rho_{0}}(x, \epsilon, \alpha, t)\right| & =\left|D_{1} g(x, \epsilon, t) \chi\left(x / \rho_{0}\right)+\frac{1}{\rho_{0}} g(x, \epsilon, t) D \chi\left(x / \rho_{0}\right)\right| \\
& \leq \frac{\delta}{2}+\frac{1}{\rho_{0}} \cdot \frac{\delta \rho_{0}}{2 A} \cdot A=\delta .
\end{aligned}
$$

The same result holds trivially when $|x| \geq 2 \rho_{0}$ since then $g_{\rho_{0}}(x, \epsilon, \alpha, t)=0$.

We now show that it is possible to use a single space, $\mathbb{Z}_{\eta_{0}}$, for our present purposes using a proof motivated by the stable manifold theorem. Part of the statement of the standard stable manifold theorem is, roughly speaking, if $x=0$ is a hyperbolic equilibrium for a differential equation and if $\varphi$ is a solution with $\sup _{t \geq t_{0}}|\varphi(t)|$ sufficiently small, then $\varphi(t) \rightarrow 0$ as $t \rightarrow+\infty$. In fact, the same proof works if one requires only that $\sup _{t \geq t_{0}}|\varphi(t)| e^{-\eta t}$ be sufficiently small for appropriate $\eta>0$. The following proof is adapted from the proof of Theorem 4.1 in Ch. 13 of Coddington and Levinson [2]. 
4. Theorem. There exist positive constants $\eta_{0}, \rho_{0}, \epsilon_{0}, \delta$ such that the following holds: if in (8) we have $\rho=\rho_{0},|\epsilon| \leq \epsilon_{0}$ and if $\psi \in \mathbb{Z}_{\eta_{0}}$ is a solution to (8) with $\|\psi\|_{\eta_{0}}<\delta$, then $\psi$ satisfies $\sup _{t}|\psi(t)| e^{\eta_{0}|t|}<\infty$ and $\sup _{t}|\psi(t)| \leq \rho_{0}$ so that $\psi$ is a homoclinic solution to (6).

Proof. As the eigenvalues of $D f_{0}(0)$ lie off the imaginary axis, we can choose $\eta_{0}>0$ such that $|\Re(\lambda)| \geq 3 \eta_{0}$ for each eigenvalue, $\lambda$, of $D f_{0}(0)$. Let $\mathcal{B}$ denote a closed ball which contains the orbit $\gamma$. We can choose $C>0$ so that $|\gamma(t)| \leq C e^{-\eta_{0} t}$ and

$$
\sup _{|\epsilon| \leq 1} \sup _{x \in \mathcal{B}}\left|D_{1} f_{1}(x, \epsilon, \epsilon t+\alpha)\right| \leq C .
$$

Furthermore, the equation $\dot{v}=D f_{0}(0) v$ has a fundamental solution $V(t)=e^{t D f_{0}(0)}$ along with projections $P_{1}, P_{2}$ and constants $A>0, \eta_{0}>0$ such that $P_{1}+P_{2}=I$ and

$$
\begin{array}{ll}
\left|V(t) P_{1} V(s)^{-1}\right| \leq A e^{2 \eta_{0}(s-t)} & \text { for } s \leq t, \\
\left|V(t) P_{2} V(s)^{-1}\right| \leq A e^{2 \eta_{0}(t-s)} & \text { for } t \leq s .
\end{array}
$$

We now write (8) in the form

$$
\dot{z}(t)=D f_{0}(0) z(t)+h_{\rho}(z(t), \epsilon, t)
$$

where

$$
h_{\rho}(x, \epsilon, \alpha, t)=\left[D f_{0}(\gamma(t))-D f_{0}(0)\right] x+g_{\rho}(x, \epsilon, \alpha, t) .
$$

Let $K_{0}=\sup _{t}\left|D f_{0}(\gamma(t))-D f_{0}(0)\right|$. Using Lemma 3 we choose $\rho_{0}>0, \epsilon_{1}>0$ such that

$$
\left|D_{1} g_{\rho_{0}}(x, \epsilon, \alpha, t)\right| \leq \min \left\{\frac{3 \eta_{0}}{16 A}, K_{0}\right\} \quad \text { for }|\epsilon| \leq \epsilon_{1} .
$$

Define $\epsilon_{0}=\min \left\{\epsilon_{1}, 1, \frac{3 \rho_{0} \eta_{0}}{16 A C^{2}}\right\}$.

Next, we choose $t_{0}>0$ so that

$$
\left|D f_{0}(\gamma(t))-D f_{0}(0)\right| \leq \min \left\{\frac{3 \eta_{0}}{16 A}, K_{0}\right\} \quad \text { for } t \geq t_{0} .
$$

Combining these two results we get

$$
\left|D_{1} h_{\rho_{0}}(x, \epsilon, \alpha, t)\right| \leq \frac{3 \eta_{0}}{8 A} \quad \text { for } t \geq t_{0},|\epsilon| \leq \epsilon_{1} .
$$

Fix $x_{1}, x_{2} \in \mathbb{R}^{n}$ and for $s \in[0,1]$ define $\phi(s)=g_{\rho_{0}}\left(s x_{1}+(1-s) x_{2}, \epsilon, \alpha, t\right)$. Then we have

$$
\begin{aligned}
g_{\rho_{0}}\left(x_{1}, \epsilon, \alpha, t\right)- & g_{\rho_{0}}\left(x_{2}, \epsilon, \alpha, t\right)=\phi(1)-\phi(0) \\
=\int_{0}^{1} \phi^{\prime}(s) d s & =\int_{0}^{1} D_{1} g_{\rho_{0}}\left(s x_{1}+(1-s) x_{2}, \epsilon, \alpha, t\right)\left(x_{1}-x_{2}\right) d s .
\end{aligned}
$$

Taking norms we get

$$
\left|g_{\rho_{0}}\left(x_{1}, \epsilon, \alpha, t\right)-g_{\rho_{0}}\left(x_{2}, \epsilon, \alpha, t\right)\right| \leq K_{0}\left|x_{1}-x_{2}\right| \quad \text { for }|\epsilon| \leq \epsilon_{0} .
$$

In a similar way we obtain

$$
\left|h_{\rho_{0}}\left(x_{1}, \epsilon, \alpha, t\right)-h_{\rho_{0}}\left(x_{2}, \epsilon, \alpha, t\right)\right| \leq \frac{3 \eta_{0}}{8 A}\left|x_{1}-x_{2}\right| \quad \text { for }|\epsilon| \leq \epsilon_{0}, t \geq t_{0}
$$


and

$$
\left|h_{\rho_{0}}(0, \epsilon, \alpha, t)\right|=\left|\epsilon f_{1}(\gamma(t), \epsilon, \epsilon t+\alpha)\right| \leq \frac{3 \rho_{0} \eta_{0}}{16 A} e^{-\eta_{0} t} \quad \text { for }|\epsilon| \leq \epsilon_{0}, t \geq 0 .
$$

Finally, choose $\delta>0$ so that

$$
\delta \leq \min \left\{\frac{\rho_{0}}{4 A} e^{-3 \eta_{0} t_{0}}, \frac{3 \rho_{0}}{8 A}, \frac{9 \rho_{0} \eta_{0}}{64 A K_{0}} e^{-\eta_{0} t_{0}}\right\} .
$$

In (8) fix $\rho=\rho_{0}$ and also fix $(\epsilon, \alpha)$ with $|\epsilon| \leq \epsilon_{0}$. Let $\psi \in \mathbb{Z}_{\eta_{0}}$ be a solution to (8) with $\|\psi\|_{\eta_{o}} \leq \delta$. For any $a \in \mathbb{R}$ we have

$$
\begin{aligned}
\psi(t)=V(t) V(a)^{-1} \psi(a)+V(t) \int_{a}^{t} V(s)^{-1} h_{\rho_{0}}(\psi(s), \epsilon, \alpha, s) d s \\
=V(t) P_{1} V(a)^{-1} \psi(a)+V(t) \int_{a}^{t} P_{1} V(s)^{-1} h_{\rho_{0}}(\psi(s), \epsilon, \alpha, s) d s \\
\quad-V(t) \int_{t}^{\infty} P_{2} V(s)^{-1} h_{\rho_{0}}(\psi(s), \epsilon, \alpha, s) d s \\
+V(t) P_{2}\left[V(a)^{-1} \psi(a)+\int_{a}^{\infty} P_{2} V(s)^{-1} h_{\rho_{0}}(\psi(s), \epsilon, \alpha, s) d s\right] .
\end{aligned}
$$

In this last equation each term grows no faster than $e^{\eta_{0} t}$ except the last whose behavior is $e^{2 \eta_{0} t}$ as $t \rightarrow+\infty$. Consequently, the expression in square brackets is zero and we have

$$
\begin{aligned}
\psi(t)=V(t) P_{1} V(a)^{-1} \psi(a) & +V(t) \int_{a}^{t} P_{1} V(s)^{-1} h_{\rho_{0}}(\psi(s), \epsilon, \alpha, s) d s \\
& -V(t) \int_{t}^{\infty} P_{2} V(s)^{-1} h_{\rho_{0}}(\psi(s), \epsilon, \alpha, s) d s .
\end{aligned}
$$

Define a Banach space, $\mathbb{X}$, by

$$
\mathbb{X}=\left\{\varphi \in \mathcal{C}^{0}\left(\left[t_{0}, \infty\right), \mathbb{R}^{n}\right): \sup _{t \geq t_{0}}|\varphi(t)| e^{\eta_{0} t}<\infty\right\}
$$

with norm, $\|\cdot\|_{\mathbb{X}}$, the supremum in the definition. We shall prove the existence of a solution, $\varphi_{0} \in \mathbb{X}$, to (13) and then show that, necessarily, $\varphi_{0}=\psi$.

Let $B_{\rho_{0}}$ be the open ball in $\mathbb{X}$ with center at the origin, radius $\rho_{0}$ and define the $\mathcal{C}^{1} \operatorname{map} F: B_{\rho_{0}} \rightarrow \mathbb{X}$ by

$$
\begin{aligned}
F(\varphi)(t)=V(t) P_{1} V\left(t_{0}\right)^{-1} \psi\left(t_{0}\right) & +V(t) \int_{t_{0}}^{t} P_{1} V(s)^{-1} h_{\rho_{0}}(\varphi(s), \epsilon, \alpha, s) d s \\
& -V(t) \int_{t}^{\infty} P_{2} V(s)^{-1} h_{\rho_{0}}(\varphi(s), \epsilon, \alpha, s) d s .
\end{aligned}
$$

The fixed points of $F$ are solutions in $B_{\rho_{0}}$ of (13) with $a=t_{0}$.

Using (11) we get $\left\|F\left(\varphi_{1}\right)-F\left(\varphi_{2}\right)\right\|_{\mathbb{X}} \leq \frac{1}{2}\left\|\varphi_{1}-\varphi_{2}\right\|_{\mathbb{X}}$ and from (12) we have $\|F(0)\|_{\mathbb{X}} \leq \frac{1}{2} \rho_{0}$. Thus, by the contraction mapping theorem (see e.g. Theorem 10.1.2 of [4]), there exists a fixed point, and hence a solution to (13), $\varphi_{0} \in B_{\rho_{0}}$ defined for $t \geq t_{0}$.

Now let $B=\sup _{t \geq t_{0}}\left|\varphi_{0}(t)-\psi(t)\right| e^{-\eta_{0} t}$. Using the fact that $\varphi_{0}$ and $\psi$ both satisfy (13) with $a=t_{0}$ along with (11) we get $\left|\varphi_{0}(t)-\psi(t)\right| \leq \frac{1}{2} B e^{\eta_{0} t}$ for $t \geq t_{0}$ and from this $B \leq \frac{1}{2} B$. But this means $B=0$ so then $\psi(t)=\varphi_{0}(t)$ for $t \geq t_{0}$. 
In particular, this implies that $\psi(t)$ goes to zero like $e^{-\eta_{0} t}$ as $t \rightarrow \infty$. A similar argument holds as $t \rightarrow-\infty$.

Now consider $0 \leq t \leq t_{0}$. Combining the definition of $K_{0},(10)$ and (12) we have

$$
\left|h_{\rho_{0}}(\psi(t), \epsilon, \alpha, t)\right| \leq 2 K \delta e^{\eta_{0} t}+\frac{3 \eta_{0} \rho_{0}}{16 A} e^{-\eta_{0} t} .
$$

Substituting this result into (13) with $a=0$ we get $|\psi(t)| \leq \rho_{0}$. A similar argument holds for $-t_{0} \leq t \leq 0$. Thus $|\psi(t)| \leq \rho_{0}$ for all $t$ so that $\psi$ is a solution to (6).

The solution of (8) is equivalent to solving the two equations

$$
\begin{aligned}
& \dot{z}=D f_{0}(\gamma) z+(I-\Pi) G_{\rho}(z, \epsilon, \alpha), \\
& \Pi\left(G_{\rho}(z, \epsilon, \alpha)\right)=0 .
\end{aligned}
$$

The proof of our main result proceeds by solving (14a) for $z$. This is achieved by the following variant of the contraction mapping principle.

5. Theorem. Let $X, Y, Z$ be Banach spaces with $U \subset X, V \subset Y$ open neighborhoods of the respective origins. Let $F: U \times V \times Z \rightarrow X$, denoted $(x, y, z) \rightarrow$ $F(x, y, z)$, be a $\mathcal{C}^{1}$ map satisfying $F(0,0, z)=0$. Suppose, further, that there exist closed neighborhoods of the respective origins $\tilde{U} \subset U, \tilde{V} \subset V$ with nonempty interior such that $\left|D_{1} F(x, y, z)\right| \leq \lambda<1$ and $D_{2} F(x, y, z)$ is bounded on $\tilde{U} \times \tilde{V} \times Z$. Then, there exist an open ball about the origin, $B_{\delta}(0) \subset \tilde{V}$, and a $\mathcal{C}^{1}$ function $\psi: B_{\delta}(0) \times Z \rightarrow X$ such that $\psi(0, z)=0$ and $F(\psi(y, z), y, z)=\psi(y, z)$. Furthermore, $\psi(y, z)$ and $D_{1} \psi(y, z)$ are bounded for $y \in B_{\delta}(0), z \in Z$.

Proof. Let $B_{r}(0) \subset \tilde{U}, B_{\delta}(0) \subset \tilde{V}$ be open balls of radii $r, \delta$ respectively centered at the respective origins with $\bar{B}_{r}(0), \bar{B}_{\delta}(0)$ their respective closures. By hypothosis $F: \bar{B}_{r}(0) \times \bar{B}_{\delta}(0) \times Z \rightarrow \bar{B}_{r}(0)$ is a uniform contraction.

By Theorem 3.2 in $\S 0.3$ of Hale [8] there exists a $\mathcal{C}^{1}$ function $\psi: B_{\delta}(0) \times Z \rightarrow$ $B_{r}(0)$ such that $F(\psi(y, z), y, z)=\psi(y, z)$. In particular, $\|\psi(y, z)\| \leq r$. Since $\left\|D_{1} F(\psi(y, z), y, z)\right\| \leq \lambda$, the linear function $I-D_{1} F(\psi(y, z), y, z)$ is invertible with norm less than or equal to $1 /(1-\lambda)$. By implicit differentiation we have

$$
D_{1} \psi(y, z)=\left(I-D_{1} F(\psi(y, z), y, z)\right)^{-1} D_{2} F(\psi(y, z), y, z) .
$$

This formula shows that $D_{1} \psi(y, z)$ is bounded.

We now come to the first of our main results. The following theorem gives the existence of homoclinic solutions for (2).

6. Theorem. There exist $\eta_{0}>0$, a connected open set $V \subset \mathbb{R} \times \mathbb{R}^{d-1}$ with $(0,0) \in$ $V$ and $\mathcal{C}^{2}$ functions $H: V \times \mathbb{R} \rightarrow \mathbb{R}^{d}, \Gamma: V \times \mathbb{R} \rightarrow \mathbb{Z}_{\eta_{0}}$ denoted $H(\epsilon, \beta, \alpha), \Gamma(\epsilon, \beta, \alpha)$ with the following properties:

(i) If $H(\epsilon, \beta, \alpha)=0$, then $\Gamma(\epsilon, \beta, \alpha)$ is a homoclinic solution to (2),

(ii) $\Gamma(0,0, \alpha)=\gamma$,

(iii) $\frac{\partial \Gamma}{\partial \beta_{j}}(0,0, \alpha)=u_{j+d}$,

(iv) $H(0,0, \alpha)=0$

(v) $\frac{\partial H_{i}}{\partial \epsilon}(0,0, \alpha)=\int_{-\infty}^{\infty}\left\langle u_{i}^{\perp}(t), f_{1}(\gamma(t), 0, \alpha)\right\rangle d t$

(vi) $\frac{\partial H_{i}}{\partial \beta_{j}}(0,0, \alpha)=0$, 
(vii) $\frac{\partial^{2} H_{i}}{\partial \beta_{j} \partial \beta_{k}}(0,0, \alpha)=\int_{-\infty}^{\infty}\left\langle u_{i}^{\perp}, D^{2} f_{0}(\gamma) u_{j+d} u_{k+d}\right\rangle d t$.

Proof. Let $\eta_{0}, \rho_{0}, \epsilon_{0}$ and $\delta$ be as in Theorem 4 and fix $\rho=\rho_{0}$ in (8). Now define the $\mathcal{C}^{1}$ function $F: \mathbb{Z}_{\eta_{0}} \times \mathbb{R} \times \mathbb{R}^{d-1} \times \mathbb{R} \rightarrow \mathbb{Z}_{\eta_{0}}$ by

$$
F(z, \epsilon, \beta, \alpha)=\sum_{j=1}^{d-1} \beta_{j} u_{d+j}+K\left((I-\Pi) G_{\rho_{0}}(z, \epsilon, \alpha)\right) .
$$

The fixed points of $F$ are solutions in $\mathbb{Z}_{\eta_{0}}$ to (14a) satisfying $\langle z(0), \dot{\gamma}(0)\rangle=0$.

From (7a) we have $F(0,0,0, \alpha)=0$ and, from (7b), $D_{1} F(0,0,0, \alpha)=0$. Using Lemma 3 we can assume that $\rho_{0}$ and $\epsilon_{0}$ have been chosen small enough so that $\left\|D_{1} F(z, \epsilon, \beta, \alpha)\right\|_{\eta_{0}} \leq 1 / 2$ for $|\epsilon| \leq \epsilon_{0}$. By assumption (v) for (1) the necessary derivatives of $F$ with respect to $(z, \beta)$ are bounded uniformly in $\alpha$ so that Theorem 5 applies. This yields an open neighborhood $V \subset \mathbb{R} \times \mathbb{R}^{d-1}$ and a $\mathcal{C}^{1}$ function $\psi: V \times \mathbb{R} \rightarrow \mathbb{Z}_{\eta_{0}}$ denoted $(\epsilon, \beta, \alpha) \rightarrow \psi(\epsilon, \beta, \alpha)$ such that $\psi(0,0, \alpha)=0$, the derivatives $D_{1} \psi(\epsilon, \beta, \alpha)$ and $D_{2} \psi(\epsilon, \beta, \alpha)$ are bounded for $(\epsilon, \beta, \alpha) \in V \times \mathbb{R}$, and

$$
\psi(\epsilon, \beta, \alpha)=\sum_{j=1}^{d-1} \beta_{j} u_{d+j}+K\left((I-\Pi) G_{\rho_{0}}(\psi(\epsilon, \beta, \alpha), \epsilon, \alpha)\right) .
$$

Using the uniform boundedness of $D_{1} \psi$ and $D_{2} \psi$ we can, by taking $V$ smaller if necessary, assume $\|\psi(\epsilon, \beta, \alpha)\|_{\eta_{0}} \leq \delta$ for $(\epsilon, \beta, \alpha) \in V \times \mathbb{R}$. But then, by Theorem 4 ,

$$
\sup _{t}|\psi(\epsilon, \beta, \alpha)(t)| e^{-\eta_{0}|t|}<\infty .
$$

From the hypothosis $f_{1}(0, \epsilon, t)=0$ it follows that $D_{3} f_{1}(0, \epsilon, t)=0$ and then that

$$
\sup _{t}\left|D_{3} f_{1}(\gamma(t)+\psi(\epsilon, \beta, \alpha)(t), \epsilon, t)\right| e^{-\eta_{0}|t|}<\infty .
$$

Differentiating (15) and using (16) and (17) we see that $(\partial \psi / \partial \epsilon)(\epsilon, \beta, \alpha)(t)$ has the asymptotic behavior $t e^{-\eta_{0}|t|}$. Proceeding in a similar way we find that the derivative $\left(\partial^{2} \psi / \partial \epsilon^{2}\right)(\epsilon, \beta, \alpha)(t)$ behaves like $t^{2} e^{-\eta_{0}|t|}$. Thus $\psi$ is $\mathcal{C}^{2}$.

Differentiating (15) and using (7b) we get

$$
\frac{\partial \psi}{\partial \beta_{j}}(0,0, \alpha)=u_{d+j} .
$$

The conditions for a solution to (8) are that $\psi$ be a solution to (14b). These conditions are $\Pi\left(G_{\rho_{0}}(\psi(\epsilon, \beta, \alpha), \epsilon, \alpha)\right)=0$ or, equivalently, $H(\epsilon, \beta, \alpha)=0$ where

$$
H_{i}(\epsilon, \beta, \alpha)=\int_{-\infty}^{\infty}\left\langle u_{i}^{\perp}(t), G_{\rho_{0}}(\psi(\epsilon, \beta, \alpha), \epsilon, \alpha)(t)\right\rangle d t, \quad 1 \leq i \leq d .
$$

If $H(\epsilon, \beta, \alpha)=0$, then $\psi(\epsilon, \beta, \alpha)$ is a solution to (6) by Theorem 4 and then $\Gamma(\epsilon, \beta, \alpha)=\psi(\epsilon, \beta, \alpha)+\gamma$ is a solution to (2). This proves (i). Part (ii) comes from $\psi(0,0, \alpha)=0$, part (iii) from (18). Part (iv) is obtained from $\psi(0,0, \alpha)=0$ and (7a). The remaining parts are obtained by differentiation of the preceding formula along with (7) and (18). 
Motivated by the preceding theorem we are led to make the following definitions for $\alpha \in \mathbb{R}, \epsilon \in \mathbb{R}, \beta \in \mathbb{R}^{d-1}$.

$$
\begin{aligned}
& a_{i}(\alpha)=\frac{\partial H_{i}}{\partial \epsilon}(0,0, \alpha)=\int_{-\infty}^{\infty}\left\langle u_{i}^{\perp}(t), f_{1}(\gamma(t), 0, \alpha)\right\rangle d t, \quad 1 \leq i \leq d, \\
& b_{i j k}=\frac{\partial^{2} H_{i}}{\partial \beta_{k} \partial \beta_{j}}(0,0, \alpha)=\int_{-\infty}^{\infty}\left\langle u_{i}^{\perp}, D^{2} f_{0}(0) u_{j+d} u_{k+d}\right\rangle d t \quad\left\{\begin{array}{c}
1 \leq i \leq d, \\
1 \leq j, k \leq d-1,
\end{array}\right. \\
& M_{i}(\epsilon, \beta, \alpha)=a_{i}(\alpha) \epsilon+\frac{1}{2} \sum_{j, k=1}^{d-1} b_{i j k} \beta_{j} \beta_{k}, \quad 1 \leq i \leq d .
\end{aligned}
$$

The conditions for the existence of a homoclinic solution are

$$
H(\epsilon, \beta, \alpha)=M(\epsilon, \beta, \alpha)+\ldots=0 .
$$

Our next result shows that it is sufficient to solve the equation $M(\epsilon, \beta, \alpha)=0$.

Geometrically, the function $H$ can be interpreted as representing the distance between the stable and unstable manifolds for the perturbed equations. When these manifolds meet in dimension one $(d=1)$, the terms of $H$ linear in $\epsilon$ are sufficient, along with the implicit function theorem. When the manifolds have a mutual tangent space of dimension greater than one $(d>1)$, the linear terms do not discriminate between the manifolds and one must look at their curvatures. The complications which this produces in the geometric approach are eliminated by the function space approach used here.

7. Theorem. Let $\dot{x}=f_{0}(x)+\epsilon f_{1}(x, \epsilon, \epsilon t+\alpha)$ be as in (2), $H$ as in Theorem 6 and $M$ as above. If $M\left(\epsilon_{0}, \beta_{0}, \alpha_{0}\right)=0$ and $D_{(\beta, \alpha)} M\left(\epsilon_{0}, \beta_{0}, \alpha_{0}\right)$ is nonsingular, then there exist an open interval, $J$, containing zero and differentiable functions $\psi: J \rightarrow \mathbb{R}^{d-1}$, $\phi: J \rightarrow \mathbb{R}$ with $\psi(0)=0, \phi(0)=0$ such that $H\left(s^{2} \epsilon_{0}, s\left(\beta_{0}+\psi(s)\right), \alpha_{0}+\phi(s)\right)=0$ for $s \in J$.

In particular, this implies that for $s \in J$ the equation

$$
\dot{x}=f_{0}(x)+s^{2} \epsilon_{0} f_{1}\left(x, s^{2} \epsilon_{0}, s^{2} \epsilon_{0} t+\alpha_{0}+\phi(s)\right)
$$

has a homoclinic solution, $\gamma_{s}, \mathcal{C}^{2}$ in $s$, satisfying $\gamma_{0}=\gamma$ and

$$
\left.\frac{\partial \gamma_{s}}{\partial s}\right|_{s=0}=\sum_{k=1}^{d-1} \beta_{0, k} u_{k+d}
$$

Proof. Define the function $F: \mathbb{R}^{d-1} \times \mathbb{R} \times \mathbb{R} \rightarrow \mathbb{R}^{d-1}$ by

$$
F_{i}(x, y, s)= \begin{cases}\frac{1}{s^{2}} H_{i}\left(s^{2} \epsilon_{0}, s\left(\beta_{0}+x\right), \alpha_{0}+y\right), & \text { for } s \neq 0 \\ M\left(\epsilon_{0}, \beta_{0}+x, \alpha_{0}+y\right), & \text { for } s=0\end{cases}
$$

We have $F(0,0,0)=0$ and $D_{(x, y)} F(0,0,0)=D_{(\beta, \alpha)} M\left(\epsilon_{0}, \beta_{0}, \alpha_{0}\right)$. The result now follows from the implicit function theorem and Theorem 6 setting

$$
\gamma_{s}=\Gamma\left(s^{2} \epsilon_{0}, s\left(\beta_{0}+\psi(s)\right), \alpha_{0}+\phi(s)\right) .
$$

8. Theorem. Suppose that, in (1b), $f_{1}$ is periodic in $t$. Let the function $M$ be as above, suppose that $M\left(\epsilon_{0}, \beta_{0}, \alpha_{0}\right)=0$ and that $D_{(\beta, \alpha)} M\left(\epsilon_{0}, \beta_{0}, \alpha_{0}\right)$ is nonsingular. Then there exists $\delta>0$ such that if $\epsilon \in(-\delta, 0)$ when $\epsilon_{0}<0$ or $\epsilon \in(0, \delta)$ when $\epsilon_{0}>0$, then the period map for (1a) has a transverse homoclinic point and hence exhibits chaos. 
Proof. Let $\phi: J \rightarrow \mathbb{R}$ and $\gamma_{s}$ be as in Theorem 7 . The variational equation along $\gamma_{s}$ is

$$
\dot{u}=\left[D f_{0}(\gamma)+S(s)\right] u
$$

where

$$
S(s)(t)=D f_{0}\left(\gamma_{s}(t)\right)-D f_{0}(\gamma(t))+s^{2} \epsilon_{0} D_{1} f_{1}\left(\gamma_{s}(t), s^{2} \epsilon_{0}, s^{2} \epsilon_{0} t+\alpha_{0}+\phi(s)\right) .
$$

We need to show that (19) has no nonzero solution which decays at both $\pm \infty$. For this we use Theorem 2.

Let $h=\left.\left(\partial \gamma_{s} / \partial s\right)\right|_{s=0}$. From Theorem $7, h=\sum_{k=1}^{d-1} \beta_{0, k} u_{k+d}$. Note that

$$
S(0)=0, \quad S^{\prime}(0)=D^{2} f_{0}(\gamma) h .
$$

In the notation of Theorem 2 we let $Q(s)=\mathcal{F}(S(s))$ and write $Q(s)=\left[q_{i j}(s)\right]$. We must show that $Q(s)$ is nonsingular for sufficiently small nonzero $|s|$.

First we have

$$
Q(0)=0
$$

Next we compute

$$
\begin{aligned}
q_{i j}^{\prime}(0) & =\int_{-\infty}^{\infty}\left\langle u_{i}^{\perp}, S^{\prime}(0) u_{j}\right\rangle d t \\
& =\int_{-\infty}^{\infty}\left\langle u_{i}^{\perp}, D^{2} f_{0}(\gamma) h u_{j+d}\right\rangle d t \\
& =\sum_{k=1}^{d-1} \beta_{0, k} \int_{-\infty}^{\infty}\left\langle u_{i}^{\perp}, D^{2} f_{0}(\gamma) u_{k+d} u_{j+d}\right\rangle d t .
\end{aligned}
$$

From this last equation we get

$$
q_{i j}^{\prime}(0)=\sum_{k=1}^{d-1} b_{i j k} \beta_{0, k}, \quad 1 \leq i \leq d, \quad 1 \leq j \leq d-1 .
$$

We need a separate calculation for $q_{i d}$. That $\gamma_{s}$ is a solution to the perturbed equation means

$$
\dot{\gamma}_{s}(t)=f_{0}\left(\gamma_{s}(t)\right)+s^{2} \epsilon_{0} f_{1}\left(\gamma_{s}(t), s^{2} \epsilon_{0}, s^{2} \epsilon_{0} t+\alpha_{0}+\phi(s)\right) .
$$

Differentiating the preceding equation yields

$$
\ddot{\gamma}_{s}=D f_{0}(\gamma) \dot{\gamma}_{s}+\varphi_{s}
$$

where

$$
\varphi_{s}(t)=S(s)(t) \dot{\gamma}_{s}(t)+s^{4} \epsilon_{0}^{2} D_{3} f_{1}\left(\gamma_{s}(t), s^{2} \epsilon_{0}, s^{2} \epsilon_{0} t+\alpha_{0}+\phi(s)\right)
$$

and from (5) and (22)

$$
\Pi \varphi_{s}=0 .
$$

Consider the equation $\dot{u}=D f_{0}(\gamma) u+\varphi_{s}$. From (22) we know that $\dot{\gamma}_{s}$ is a solution to this equation. Using the property of the variation of constants map, $K$, defined above and (23) we have

$$
\dot{\gamma}_{s}=K \varphi_{s}+U P_{s s} v(s)=K(I-\Pi) \varphi_{s}+U P_{s s} v(s)
$$


for some $\mathcal{C}^{2}$ function $v: J \rightarrow \mathbb{R}^{n}$. The entries of $v$ other than $v_{d+1}, \ldots, v_{2 d}$ are arbitrary and can be taken as zero. Evaluating the preceding equation at $s=0$ yields $U P_{s s} v(0)=\dot{\gamma}$ and by substituting this we get an equation of the form

$$
\dot{\gamma}_{s}=K(I-\Pi) S(s) \dot{\gamma}_{s}+\dot{\gamma}+s \sum_{k=1}^{d} w_{k+d}(s) u_{k+d}+O\left(s^{4}\right)
$$

where $w(s)=(v(s)-v(0)) / s$.

Choose $\delta_{1}>0$ so that when $|s|<\delta_{1}, I-K(I-\Pi) \hat{S}(s)$ is invertible. Then for $s$ in this range the preceding equation can be solved for $\dot{\gamma}_{s}$ to yield

$$
[I-K(I-\Pi) \hat{S}(s)]^{-1} \dot{\gamma}=\dot{\gamma}_{s}-s \sum_{k=1}^{d} w_{k+d}(s)[I-K(I-\Pi) \hat{S}(s)]^{-1} u_{k+d}+O\left(s^{4}\right)
$$

and, using (22),

$$
\begin{aligned}
\hat{S}(s)[I- & K(I-\Pi) \hat{S}(s)]^{-1} \dot{\gamma} \\
= & S(s) \dot{\gamma}_{s}-s \sum_{k=1}^{d} w_{k+d}(s) \hat{S}(s)[I-K(I-\Pi) \hat{S}(s)]^{-1} u_{k+d}+O\left(s^{5}\right) \\
= & \ddot{\gamma}_{s}-D f_{0}(\gamma) \dot{\gamma}_{s}-s^{4} \epsilon_{0}^{2} D_{3} f_{1}\left(\gamma, 0, \alpha_{0}\right) \\
& \quad-s \sum_{k=1}^{d} w_{k+d}(s) \hat{S}(s)[I-K(I-\Pi) \hat{S}(s)]^{-1} u_{k+d}+O\left(s^{5}\right) .
\end{aligned}
$$

Next, using (4),

$$
\begin{aligned}
q_{i d}(s) & =\int_{-\infty}^{\infty}\left\langle u_{i}^{\perp}, \hat{S}(s)[I-K(I-\Pi) \hat{S}(s)]^{-1} \dot{\gamma}\right\rangle d t \\
& =-s^{4} \epsilon_{0}^{2} \int_{-\infty}^{\infty}\left\langle u_{i}^{\perp}, D_{3} f_{1}\left(\gamma, 0, \alpha_{0}\right)\right\rangle d t-s \sum_{k=1}^{d} w_{k+d}(s) q_{i k}(s)+O\left(s^{5}\right) \\
& =-s^{4} \epsilon_{0}^{2} a_{i}^{\prime}\left(\alpha_{0}\right)-s \sum_{k=1}^{d} w_{k+d}(s) q_{i k}(s)+O\left(s^{5}\right) .
\end{aligned}
$$

Combining (20), (21) and (24) we have

$$
\operatorname{det}(Q(s))=-s^{d+3} \epsilon_{0}^{2} \operatorname{det}\left(D_{(\beta, \alpha)} M\left(\epsilon_{0}, \beta_{0}, \alpha_{0}\right)\right)+O\left(s^{d+4}\right) .
$$

From this last equation there exists $\delta_{2}$ with $0<\delta_{2} \leq \delta_{1}$ such that $Q(s)$ is nonsingular when $0<|s|<\delta_{2}$. Then by Theorem 2, (19) has an exponential dichotomy valid for all $t$. Following [10] we know that (2) has a transverse homoclinic orbit for $\epsilon=s^{2} \epsilon_{0}, \alpha=\alpha_{0}+\phi(s)$. Then the period map for (1b) has a transverse homoclinic point for $\epsilon \in(-\delta, 0)$ when $\epsilon_{0}<0$ or $\epsilon \in(0, \delta)$ when $\epsilon_{0}>0$ where $\delta=\delta_{2}\left|\epsilon_{0}\right|$.

Let us look at some special cases of the preceding theorem. If $x \in \mathbb{R}^{2}$, we must have $d=1$ and $u_{2}=\dot{\gamma}$. Denoting $\gamma=\left(\gamma_{1}, \gamma_{2}\right)$ we have

$$
u_{1}^{\perp}(t)=\left(-\dot{\gamma}_{2}(t), \dot{\gamma}_{1}(t)\right) \exp \left(-\int_{0}^{t}\left(\nabla \cdot f_{0}\right)(\gamma(s)) d s\right)
$$

and knowledge of $u_{1}$ is not required. There is no $\beta$ and the condition for a homoclinic solution is the scalar equation $H(\epsilon, \alpha)=0$. 
The bifurcation equation takes the form $M(\epsilon, \alpha)=a(\alpha) \epsilon=0$ so we can take $\epsilon_{0}= \pm 1$. Theorem 8 now reduces to the following.

9. Corollary. Suppose that in (1a) we have $x \in \mathbb{R}^{2}$ and $f_{1}$ periodic in $t$. Define

$$
a(\alpha)=\int_{-\infty}^{\infty} \operatorname{det}\left(\dot{\gamma}(t), f_{1}(\gamma(t), 0, \alpha)\right) \exp \left(-\int_{0}^{t}\left(\nabla \cdot f_{0}\right)(\gamma(s)) d s\right) d t .
$$

If there exists $\alpha_{0}$ such that $a\left(\alpha_{0}\right)=0$ and $a^{\prime}\left(\alpha_{0}\right) \neq 0$, then there exists $\delta>0$ such that (1a) has a transverse homoclinic orbit when $0<|\epsilon|<\delta$.

The preceding result generalizes with little difficulty to higher $n$ as long as we have $d=1$. The difference between $n=2$ and $n>2$ is that it is necessary to use the exponential dichotomy, $U$, as given in Theorem 1 . The following result is similar to the main theorem of [1].

10. Corollary. Suppose that in (1a) we have $d=1$ and that $f_{1}$ is periodic in $t$. Define

$$
a(\alpha)=\int_{-\infty}^{\infty}\left\langle u_{1}^{\perp}(t), f_{1}(\gamma(t), 0, \alpha)\right\rangle d t .
$$

If there exists $\alpha_{0}$ such that $a\left(\alpha_{0}\right)=0$ and $a^{\prime}\left(\alpha_{0}\right) \neq 0$, then there exists $\delta>0$ such that (1a) has a transverse homoclinic orbit when $0<|\epsilon|<\delta$.

Solutions homoclinic to a small solution. Let us consider differential equations of the form

$$
\epsilon \dot{x}=f_{0}(x)+\epsilon f_{1}(x, \epsilon, t)
$$

with $x \in \mathbb{R}^{n}, \epsilon \in \mathbb{R}$ and assume

(i) $f_{0}$ and $f_{1}$ are $\mathcal{C}^{3}$ in all arguments.

(ii) $f_{0}(0)=0$.

(iii) The eigenvalues of $D f_{0}(0)$ lie off the imaginary axis.

(iv) The unperturbed equation has a homoclinic solution. That is, there exists a differentiable function $t \rightarrow \gamma(t)$ such that $\lim _{t \rightarrow+\infty} \gamma(t)=\lim _{t \rightarrow-\infty} \gamma(t)=0$ and $\dot{\gamma}(t)=f_{0}(\gamma(t))$.

(v) $f_{1}(x, \epsilon, t+p)=f_{1}(x, \epsilon, t)$ for some $p>0$.

(vi) $f_{1}$ and its derivatives with respect to $(x, \epsilon)$ are bounded in $(x, \epsilon)$ uniformly in $t$.

Since (25) does not have an equilibrium at $x=0$ for $\epsilon \neq 0$ there cannot be solutions homoclinic to the origin when $\epsilon \neq 0$. However, because the origin is hyperbolic, we can have solutions homoclinic to a small bounded solution. Our next result proves the existence of this solution. We define the Banach space

$$
\mathcal{C}_{p}^{0}\left(\mathbb{R}, \mathbb{R}^{n}\right)=\left\{z \in \mathcal{C}^{0}\left(\mathbb{R}, \mathbb{R}^{n}\right): z(t+p)=z(t)\right\}
$$

with $\|z\|=\sup _{t}|z(t)|$.

Let $A=D f_{0}(0)$. The linear equation $\dot{x}=A x$ has an exponential dichotomy given by $e^{A t}$ and projections $P_{1}$ and $P_{2}$ with $P_{1}+P_{2}=I$. From this one gets an exponential dichotomy for the equation $\epsilon \dot{x}=A x$ with the same projections and fundamental solution $e^{A t / \epsilon}$. 
In other words, there exist constants $C>0, M>0$ such that

$$
\begin{array}{ll}
\left|e^{A t / \epsilon} P_{1} e^{-A s / \epsilon}\right| \leq C e^{2 M(s-t) / \epsilon} \quad \text { for } s \leq t \\
\left|e^{A t / \epsilon} P_{2} e^{-A s / \epsilon}\right| \leq C e^{2 M(t-s) / \epsilon} \quad \text { for } t \leq s
\end{array}
$$

Now consider the equation

$$
\epsilon \dot{x}=A x+w
$$

with $w \in \mathcal{C}_{p}^{0}\left(\mathbb{R}, \mathbb{R}^{n}\right)$. The general solution to $(27)$ in $\mathcal{C}_{p}^{0}\left(\mathbb{R}, \mathbb{R}^{n}\right)$ is $x=K_{\epsilon}(w)$ where

$$
K_{\epsilon}(w)(t)=\frac{1}{\epsilon} e^{A t / \epsilon} \int_{-\infty}^{t} P_{1} e^{-A s / \epsilon} w(s) d s-\frac{1}{\epsilon} e^{A t / \epsilon} \int_{t}^{\infty} P_{2} e^{-A s / \epsilon} w(s) d s .
$$

Our next result extends the preceding formula to $\epsilon=0$.

11. Lemma. Let $K_{\epsilon}$ be as above and let $w \in \mathcal{C}_{p}^{0}\left(\mathbb{R}, \mathbb{R}^{n}\right)$. Then

$$
\lim _{\epsilon \rightarrow 0}\left\|K_{\epsilon}(w)+A^{-1} w\right\|=0 .
$$

Proof. Write $K_{\epsilon}(w)(t)=\sum_{i=1}^{4} \varphi_{i}(\epsilon, t)$ where

$$
\begin{aligned}
& \varphi_{1}(\epsilon, t)=\frac{1}{\epsilon} e^{A t / \epsilon} \int_{-\infty}^{t} P_{1} e^{-A s / \epsilon} w(t) d s \\
& \varphi_{2}(\epsilon, t)=\frac{1}{\epsilon} e^{A t / \epsilon} \int_{-\infty}^{t} P_{1} e^{-A s / \epsilon}[w(s)-w(t)] d s, \\
& \varphi_{3}(\epsilon, t)=-\frac{1}{\epsilon} e^{A t / \epsilon} \int_{t}^{\infty} P_{2} e^{-A s / \epsilon} w(t) d s \\
& \varphi_{4}(\epsilon, t)=-\frac{1}{\epsilon} e^{A t / \epsilon} \int_{t}^{\infty} P_{2} e^{-A s / \epsilon}[w(s)-w(t)] d s .
\end{aligned}
$$

The expressions for $\varphi_{1}$ and $\varphi_{3}$ can be integrated to yield $\varphi_{1}(\epsilon, t)+\varphi_{3}(\epsilon, t)=$ $-A^{-1} w(t)$. It remains to show that the remaining terms go to zero.

Let $\alpha>0$ be arbitrary and let $C, M$ be as in (26). By the uniform continuity of $w$ we can choose $t_{0}$ such that $|w(s)-w(t)| \leq M \alpha / C$ for $t-t_{0} \leq s \leq t$, then choose $\delta>0$ such that $\|w\| e^{-2 M t_{0} / \epsilon} \leq \frac{M \alpha}{2 C}$ whenever $0<\epsilon \leq \delta$.

We divide the integral for $\left|\varphi_{2}(\epsilon, t)\right|$ into two parts, namely, $-\infty<s \leq t-t_{0}$ and $t-t_{0} \leq s \leq t$. Using (26) it is easy to check that each of these is less than or equal to $\alpha / 2$ so $\sup _{t}\left|\varphi_{2}(\epsilon, t)\right| \leq \alpha$. Since $\alpha$ was arbitrary, $\lim _{\epsilon \rightarrow 0} \sup _{t}\left|\varphi_{2}(\epsilon, t)\right|=0$. The same result follows for $\varphi_{4}$ in a similar fashion.

12. Theorem. There exist $\epsilon_{0}>0$ and a $\mathcal{C}^{1}$ function $\varphi:\left(-\epsilon_{0}, \epsilon_{0}\right) \rightarrow \mathcal{C}_{b}^{0}\left(\mathbb{R}, \mathbb{R}^{n}\right)$ such that $\varphi(0)=0$ and $\varphi(\epsilon)$ is a solution to (25).

Proof. Write (25) in the form

$$
\epsilon \dot{x}=A x+g(x, \epsilon, t)
$$

where $A=D f_{0}(0)$ and $g(x, \epsilon, t)=f_{0}(x)-D f_{0}(0) x+\epsilon f_{1}(x, \epsilon, t)$. Using the variation of constants map, $K_{\epsilon}$, from above we can define the $\mathcal{C}^{1}$ map

$$
F: \mathcal{C}_{p}^{0}\left(\mathbb{R}, \mathbb{R}^{n}\right) \times \mathbb{R} \rightarrow \mathcal{C}_{p}^{0}\left(\mathbb{R}, \mathbb{R}^{n}\right)
$$


by

$$
F(z, \epsilon)(t)= \begin{cases}z(t)-K_{\epsilon}(G(z, \epsilon))(t), & \text { for } \epsilon \neq 0, \\ -A^{-1} f_{0}(z(t)), & \text { for } \epsilon=0,\end{cases}
$$

where $G: \mathcal{C}_{p}^{0}\left(\mathbb{R}, \mathbb{R}^{n}\right) \times \mathbb{R} \rightarrow \mathcal{C}_{p}^{0}\left(\mathbb{R}, \mathbb{R}^{n}\right)$ is defined by $G(z, \epsilon)(t)=g(z(t), \epsilon, t)$. The zeros of $F$ are solutions to $(25)$ and we have $F(0,0)=0$ and $D_{1} F(0,0)=-I$. The existence of $\varphi$ now follows from the implicit function theorem.

Let $\varphi$ be as in Theorem 12 and make the change of variable $x=y+\varphi(\epsilon)$ in (25). After changing back to $x$ we get the equation

$$
\epsilon \dot{x}=f_{0}(x)+\epsilon \bar{f}_{1}(x, \epsilon, t)
$$

where

$$
\begin{gathered}
\bar{f}_{1}(x, \epsilon, t)=\frac{1}{\epsilon}\left[f_{0}(x+\varphi(\epsilon)(t))-f_{0}(x)\right]+f_{1}(x+\varphi(\epsilon)(t), \epsilon, t)-\dot{\varphi}(\epsilon)(t) \\
=\frac{1}{\epsilon}\left[f_{0}(x+\varphi(\epsilon)(t))-f_{0}(x)-f_{0}(\varphi(\epsilon)(t))\right] \\
\quad+f_{1}(x+\varphi(\epsilon)(t), \epsilon, t)-f_{1}(\varphi(\epsilon)(t), \epsilon, t) .
\end{gathered}
$$

From the second formula for $\bar{f}_{1}$ we have $\bar{f}_{1}(0, \epsilon, t)=0$ so Theorem 7 can be used to obtain homoclinic solutions to (28). Furthermore, this yields solutions to (25) which are homoclinic to $\varphi(\epsilon)$ in the sense that $\lim _{t \rightarrow \pm \infty}[x(t)-\varphi(\epsilon)(t)]=0$.

Note that from the first formula for $\bar{f}_{1}$ we have

$$
\bar{f}_{1}(\gamma(t), 0, \alpha)=f_{1}(\gamma(t), 0, \alpha)+D f_{0}(\gamma(t)) \frac{d \varphi}{d \epsilon}(0)(\alpha) .
$$

Using (4) we see that the definition of $M(\epsilon, \beta, \alpha)$ is the same whether one uses $f_{1}$ from (25) or $\bar{f}_{1}$ from (28). This means that Theorem 8 can be applied directly to (25). For (1), Theorem 8 yields for the period map a transverse orbit homoclinic to the origin. For (25) the fixed point at the origin for the period map is replaced by a fixed point a distance $O(\epsilon)$ from the origin provided by the periodic solution $\varphi(\epsilon)$.

\section{EXAMPLES}

We now proceed to illustrate the preceding theory with some examples. To simplify the formulas we adopt the notation $r(t)=\operatorname{sech} t$. Note that we have $\ddot{r}=r-2 r^{3}$. The following values will be needed:

$$
\int_{-\infty}^{\infty} r^{2} d t=2, \quad \int_{-\infty}^{\infty} r^{4} d t=\frac{4}{3}, \quad \int_{-\infty}^{\infty} \dot{r}^{2} d t=\frac{2}{3}
$$

Example 1. Consider the equation

$$
\ddot{x}=x-2 x^{3}+\epsilon \dot{x} \sin \epsilon t
$$

which we regard as a first order system in phase space $(x, \dot{x})$. This equation has a hyperbolic fixed point at $(0,0)$ for all sufficiently small $\epsilon$. When $\epsilon=0$, we get the familiar Duffing's equation with negative stiffness, which has two homoclinic solutions. We consider the one given by $x=r$.

In the notation of Corollary 9 we have

$$
a(\alpha)=\int_{-\infty}^{\infty} \dot{r}(t)^{2} \sin \alpha d t=\frac{2}{3} \sin \alpha .
$$


The conditions $a\left(\alpha_{0}\right)=0, a^{\prime}\left(\alpha_{0}\right)=0$ are satisfied for $\alpha_{0}=\pi$. Thus Corollary 9 applies and there exists $\delta>0$ such that for $-\delta<\epsilon<\delta$ the period map of the differential equation has a transverse homoclinic orbit.

Example 2. Consider the system of equations

$$
\begin{aligned}
& \ddot{x}=x-x^{3}-x y^{2}-\epsilon x \cos \epsilon t, \\
& \ddot{y}=y-\frac{4}{3} y^{3}-\frac{2}{3} x^{3}+\epsilon \dot{y} \sin \epsilon t .
\end{aligned}
$$

A homoclinic solution, when $\epsilon=0$, is given by $x=y=r$. We work in the phase space $(x, \dot{x}, y, \dot{y})$ and find the eigenvalues of $D f_{0}(0)$ to be $\{-1,-1,1,1\}$. Thus, the system of equations has a hyperbolic equilibrium at the origin for all sufficiently small $|\epsilon|$. Letting $u=(v, \dot{v}, w, \dot{w})$ denote a solution to the variational equation we get

$$
\begin{aligned}
\ddot{v} & =v-4 r^{2} v-2 r^{2} w \\
\ddot{w} & =w-4 r^{2} w-2 r^{2} v
\end{aligned}
$$

One solution is given by $v=w=\dot{r}$ and variation of parameter leads to a second of the form $v=w=P \dot{r}$ where $P$ is a differentiable function which satisfies $\dot{r}(P \dot{r})^{\cdot}-P \ddot{r} \ddot{r}=\dot{P} \dot{r}^{2}=1$, an arbitrary constant. A third solution is given by $v=-w=r$ and, once again turning to variation of parameter, we get a fourth solution $v=-w=Q r$ where $Q$ satisfies $r(Q r)^{*}-Q r \dot{r}=\dot{Q} r^{2}=1$.

These four solutions yield a solution, $U$, as given in Theorem 1 with the projections arranged as required:

$$
U=\left[\begin{array}{cccc}
Q r & P \dot{r} & r & \dot{r} \\
(Q r)^{\cdot} & (P \dot{r})^{\cdot} & \dot{r} & \ddot{r} \\
-Q r & P \dot{r} & -r & \dot{r} \\
-(Q r)^{\circ} & (P \dot{r})^{\cdot} & -\dot{r} & \ddot{r}
\end{array}\right]
$$

These calculations show that $d=2$. We also have

$$
u_{1}^{\perp}=\frac{1}{2}\left[\begin{array}{c}
-\dot{r} \\
r \\
\dot{r} \\
-r
\end{array}\right], \quad u_{2}^{\perp}=\frac{1}{2}\left[\begin{array}{c}
-\ddot{r} \\
\dot{r} \\
-\ddot{r} \\
\dot{r}
\end{array}\right] .
$$

We now compute

$$
\begin{aligned}
a_{1}(\alpha) & =\int_{-\infty}^{\infty} \frac{1}{2} r(-r \cos \alpha)-\frac{1}{2} r(\dot{r} \sin \alpha) d t=-\cos \alpha, \\
a_{2}(\alpha) & =\int_{-\infty}^{\infty} \frac{1}{2} \dot{r}(-r \cos \alpha)+\frac{1}{2} \dot{r}(\dot{r} \sin \alpha) d t=\frac{1}{3} \sin \alpha, \\
b_{111} & =\int_{-\infty}^{\infty} \frac{1}{2} r\left(-4 r^{3}\right)-\frac{1}{2} r\left(-12 r^{3}\right) d t=\int_{-\infty}^{\infty} 4 r^{4} d t=\frac{16}{3}, \\
b_{211} & =\int_{-\infty}^{\infty} \frac{1}{2} \dot{r}\left(-4 r^{3}\right)+\frac{1}{2} \dot{r}\left(-12 r^{3}\right) d t=\int_{-\infty}^{\infty}-8 \dot{r} r^{3} d t=0 .
\end{aligned}
$$


Thus we have

$$
\begin{aligned}
M_{1}(\epsilon, \beta, \alpha) & =(-\cos \alpha) \epsilon+\frac{8}{3} \beta^{2}, \\
M_{2}(\epsilon, \beta, \alpha) & =\left(\frac{1}{3} \sin \alpha\right) \epsilon, \\
D_{(\beta, \alpha)} M(\epsilon, \beta, \alpha) & =\left[\begin{array}{cc}
\frac{16}{3} \beta & \epsilon \sin \alpha \\
0 & \frac{\epsilon}{3} \cos \alpha
\end{array}\right] .
\end{aligned}
$$

We can take $\alpha_{0}=0, \epsilon_{0}=1, \beta_{0}^{2}=\frac{3}{8}$. Theorem 8 now applies. There exists $\delta>0$ such that when $0<\epsilon<\delta$ the period map for the differential equation has a transverse homoclinic solution. We can also use $\alpha_{0}=\pi, \epsilon_{0}=-1, \beta_{0}^{2}=3 / 8$ so that, in fact, we can take $-\delta<\epsilon \delta$ for some $\delta>0$.

\section{REFERENCES}

1. F. Battelli and K. Palmer, Chaos in the Duffing Equation, J. Differential Equations 101 (1993), 276-301. MR 93k:34099

2. Earl A. Coddington and Norman Levinson, Theory of Ordinary Differential Equations, McGraw-Hill, New York, 1955. MR 16:1022b

3. W. A. Coppel, Dichotomies in Stability Theory, Lecture Notes in Mathematics, v. 629, Springer-Verlag, 1978. MR 58:1332

4. J. Dieudonné, Foundations of Modern Analysis, 2nd (enlarged and corrected) printing, Series in Pure and Applied Mathematics, v. 10-I, Academic Press, New York and London, 1969. MR 50: 1782

5. J. R. Gruendler, Homoclinic Solutions for Autonomous Dynamical Systems in Arbitrary Dimension, SIAM J. Math. Anal. 23(3) (1992), 702-721. MR 93e:34068

6. J. R. Gruendler, Homoclinic Solutions for Autonomous Ordinary Differential Equations with Nonautonomous Perturbations, J. Differential Equations 122(1) (1995), 1-26. MR 96j:58123

7. J. R. Gruendler, The Existence of Transverse Homoclinic Solutions for Higher Order Equations, J. Differential Equations 130 (1996), 307-320. MR 97g:34059

8. J. K. Hale, Ordinary Differential Equations, 2nd ed., Robert E. Krieger, New York, 1980. MR 82e:34001

9. J. Knobloch, Bifurcation of Degenerate Homoclinics in Reversible and Conservative Systems, Preprint No. M 15/94, Technical University of Ilmenau, PSF 327, D 98684 Ilmenau, Germany, 1994.

10. K. Palmer, Exponential Dichotomies and Transversal Homoclinic Points, J. Differential Equations 55 (1984), 225-256. MR 86d:58088

11. U. Schalk and J. Knobloch, Homoclinic Points Near Degenerate Homoclinics, Nonlinearity 8 (1995), 1133-1141. MR 97a:58142

12. A. Vanderbauwhede, Bifurcation of Degenerate Homoclinics, Results in Mathematics 21 (1992), 211-223. MR 93d:58131

13. A. Vanderbauwhede and S. A. Van Gils, Center Manifolds and Contractions on a Scale of Banach Spaces, J. Funct. Anal. 72 (1987), 209-224. MR 88d:58085

Department of Mathematics, North Carolina A\&t State University, Greensboro, North Carolina 27411

E-mail address: gruendlj@ncat.edu 